\title{
Espacio público y movimientos sociales: entrevista con Dr. Don Mitchell1
}

\author{
Beatriz Bustos $^{2}$ y Daniel Opazo 3
}

En el marco del programa Fulbright Specialist Program, la Escuela de Postgrado de la Facultad de Arquitectura y Urbanismo de la Universidad de Chile invitó para la inauguración del año académico a Don Mitchell, Distinguished Professor del Departamento de Geografía de la Maxwell School of Citizenship and Public Affairs, de la Universidad de Syracuse. La visita de dos semanas incluyó la realización de clases magistrales, actividades con alumnos, organizaciones sociales, visitas a terreno y la entrevista que a continuación se detalla.

DO. Quisiera comenzar recordando la actividad que tuvimos con las organizaciones sociales ${ }^{4}$. Me interesaba consultar su opinión sobre este fenómeno donde ciertos movimientos sociales y organizaciones ciudadanas se están enfocando predominantemente en materias relacionadas con el espacio, por ejemplo en el rechazo a ciertos proyectos de infraestructura o renovación urbana, todos los cuales se desarrollan dentro de un esquema de arriba hacia abajo. Si bien desde cierto punto de vista pueden ser consideradas manifestaciones NIMBY (no-en-mi-patio-trasero), al mismo tiempo desafían no solo los proyec-

\footnotetext{
1 Los autores agradecen a Fulbright Chile y la Escuela de Postgrado de la FAU por el financiamiento que permitió la visita del Prof. Mitchell.

2 Departamento de Geografía, Universidad de Chile (Chile). E-mail: bibustos@uchilefau.cl

3 Departamento de Arquitectura, Universidad de Chile. (Chile). E-mail: daniel.opazo@uchilefau.cl

4 Foro "Construcción de espacio público desde la sociedad civil" realizado el 15 de marzo de 2013 en el auditorio de la Facultad de Arquitectura y Urbanismo de la Universidad de Chile (N. del T.).
}

tos, sino también la lógica que los inspira. ¿Cree usted que esto sucede también en otros sitios o que se trata de un elemento particular de Santiago?

DM. Pienso, y subrayo el pensar porque es un término importante en este contexto, que el giro hacia la disputa espacial es sumamente amplio en muchas formas diferentes $y$ la lucha por el espacio dentro de lo que Ed Soja Ilamaría justicia espacial se ha vuelto crecientemente importante. Esta lucha tiene asimismo una larga historia, ya que estos tipos de activismo anticarreteras a veces pueden parecer NIMBY o exclusivos de clases medias altas, pero también pueden ser muy progresistas: por ejemplo, el movimiento en San Francisco en los años 70 para detener la extensión de la Embarcadero Freeway o la fallida "lucha del césped" en Glasgow para detener la extensión de la M77 alrededor de Pollok Park en los 90. Aquellas tendían a ser luchas relativamente progresistas y multifacéticas que no se trataban solamente de impedir que algo dañase equipamientos para personas de clase alta o algo así, sino que pretendían fomentar una nueva concepción respecto a lo que las ciudades son en realidad y qué implica una buena vida en ellas, que en sí mismas son preguntas profundamente espaciales. De lo poco que he visto y oído aquí, pareciera que las mismas preguntas están apareciendo y lo que se deducía de ese panel era que está emergiendo un esfuerzo para vincular ese tipo de luchas a través de los distintos barrios, a través de diferentes espacios así como también con otros tipos de movimientos y luchas. Este es un proceso siempre difícil que nunca se completa del todo satisfactoriamente. 
DO. Pregunto lo anterior porque al menos en el caso de Santiago, el espacio público siempre ha sido un centro y foco de protestas contra el gobierno nacional y también porque no contamos con un gobierno de ciudad. Es difícil para los que protestan o los participantes del movimiento identificar espacialmente el objeto de su protesta.

DM. Una de las cosas que me ha impactado desde que llegué es que la gran ventaja de los movimientos de protesta tanto en Chile como en otros lugares respecto de los Estados Unidos es el Estado centralizado, de tal manera que existe un objeto central para algunos tipos de problemas; si bien existe toda una crítica respecto a cuál sería el objeto adecuado para el movimiento estudiantil, por ejemplo, para mí es totalmente comprensible porque hay un foco constante en el Estado nacional central como un objeto. Esto también se puede ver en Francia, pensando en el Estado altamente centralizado.

BB. Pero desde otro punto de vista, el problema es que por ejemplo en comunidades rurales o alejadas del centro es mucho más difícil confrontar al Estado.

DM. Exacto, y por eso justamente iba a decir que la gran desventaja para protestas de todo tipo en Chile es el Estado altamente centralizado, es las dos cosas al mismo tiempo.

BB. De hecho, la semana pasada estaba en Cochamó entrevistando pescadores y me decían que la única forma en que podían lograr la atención del gobierno central era tomándose los caminos, quemando neumáticos en medio de la carretera para que la verdadera autoridad central deba ir allá.

DM. Y existe un tema paralelo en los EE.UU., cual es que nuestra gran ventaja es que el Estado es altamente descentralizado y a la vez nuestra gran desventaja es que el Estado es altamente descentralizado. Así, por ejemplo, los movimientos indígenas de los 90 se involucraron precisamente en ese tipo de actividades -bloqueo de caminos, quema de neumáticos en lugares marginales como las afueras de Buffalo, NY-, con el objetivo de atraer la atención de Washington hacia las reservaciones; la única forma de conseguirlo era siendo realmente militante. En los EE.UU. la dificultad de tener un sistema tan altamente descentralizado es que a menudo cuesta mucho saber dónde está el objeto (de la protesta). Sin embargo, existen diferencias en muchos lugares donde hay gobiernos municipales fuertes o al menos gobiernos municipales con mucha autoridad. Por lo tanto, para gran parte de la política a nivel de barrio, por ejemplo, existe un objetivo más claro dentro de una región. Ahora, Los Ángeles en algunos aspectos no es tan distinta de Santiago, diría yo. Hay muchísimas municipalidades en ella y los problemas son regionales. Hay temas muy locales, por supuesto, y Los Ángeles como ciudad es dominante, pero los problemas son regionales. Por ejemplo, los problemas relacionados con el financiamiento inequitativo del transporte público son un gran tema en Los Ángeles. Existe una autoridad municipal de transporte contra la cual se puede protestar, pero cuando tratas de pensar acerca de la relación entre aquello y los temas de justicia ambiental, hay tantas especificidades locales para tomar en cuenta que el asunto se vuelve un problema político muy difícil para todos los movimientos. Creo que ese es el problema que enfrenta la gente aquí, saber cuál es el objeto de protesta; es más, pienso que se ha vuelto un problema cada vez más difícil durante las dos últimas décadas y alrededor del globo ya que en tanto el Estado ha sido selectivamente debilitado, solo cuando algo grande sucede -por ejemplo el desastre de British Petroleum en el golfo de México- aparece un claro culpable que puede ser confrontado. Sin embargo, para gran parte del movimiento de capital y las inversiones bajo inversiones, o la manera en que el dinero opera para abrir espacios para nuevos desarrollos, puede ser realmente muy difícil identificar a quién se debe atacar. Y contra quienes protestan, aparecen los discursos de "ah, ustedes quieren mantener todo como está" o "ustedes están contra el libre mercado", ya saben, ese tipo de cosas. Con el debilitamiento selectivo del Estado, este no necesariamente es un buen objetivo, pero es difícil saber que sí lo es. Durante los 90 lo fueron por un tiempo firmas como Bechtel, una empresa global de ingeniería, y otras por el estilo contra las que se podían organizar boicot de consumidores. Pero de otra manera es realmente difícil saber dónde buscar. 
BB. Pero al mismo tiempo los sectores más radicales del movimiento ni siquiera intentan encontrar tal objetivo; cuando hablamos con los estudiantes, no intentan llegar al Estado porque quieren crear una alternativa completamente diferente, un nuevo tipo de sistema. Yo me pregunto de qué modo eso puede ayudar a impulsar la lucha por el espacio público, cuando pareciera formar parte de un mundo paralelo.

DM. No estoy del todo seguro, debido a que no me convencen del todo los argumentos de la horizontalidad. Eso proviene por una parte de mi propia posición política y por otra de ver experiencias en los Estados Unidos de movimientos activistas realmente efectivos, como el floreciente movimiento por el derecho a la ciudad, quienes entienden que deben enfocarse en el Estado y las particulares formas del poder estatal aunque pretendan desarrollar al interior de sus propias organizaciones estructuras más horizontales, basadas en las alianzas y coaliciones. En un lugar como Nueva York eso es un poco más fácil porque está el alcalde Bloomberg en el cargo, quien puede ser visto como un foco o un objetivo, o el rol del departamento de vivienda y conservación durante las luchas por los jardines comunitarios (community gardens), que claramente llevó adelante un conjunto de acciones en las cuales era fácil enfocarse desde el punto de vista de la comunidad movilizada. Por lo tanto, creo que debe haber maneras para desarrollar en los movimientos estructuras internas que siendo democráticas e igualitarias sean también efectivas, y del mismo modo poder realizar un análisis que permita dilucidar dónde y cómo el poder se centraliza, cuál es el rol del Estado en ello y por lo tanto qué es lo que debe ser desmantelado al interior de este. De todas maneras, pienso que pese a todos sus problemas al menos por un tiempo el movimiento del Foro Social ha sido relativamente efectivo en reunir coaliciones de base muy amplia, experimentar con otros modos de organización y también siendo muy claro respecto a lo que entienden por problemático dentro de lo que ellos enfrentan. Asimismo, pienso que el movimiento Occupy Wall Street fue, con muchas excepciones y problemas, bastante efectivo en eso también, inventando nuevas formas de toma de decisiones y distribuir poder al tiempo que instalando una serie de demandas. No siempre levantaban demandas contra el Estado y la reacción a eso era "ustedes no tienen idea alguna sobre lo que quieren hacer"; el punto es que tenían una multiplicidad de ideas. Ha habido algunas colaboraciones políticas muy interesantes en torno a Occupy, y pienso que el momento donde se hizo claro que la organización horizontal realmente podía funcionar, manteniendo el foco claro respecto a lo que perseguía y desarrollando un liderazgo orgánico fue después del huracán Sandy. Occupy Sandy se inventó el día después de la tormenta por parte de muchos activistas que trabajaron en formas muy mutualistas para llevar ayuda a partes de la ciudad que habían sido muy golpeadas por el huracán, al punto que la Cruz Roja y otras agencias comenzaron a depender de los grupos de ocupación para la distribución de agua y ropa y materiales de construcción, primeros auxilios, todo tipo de cosas, ya que la forma mutualista funcionó mucho mejor que la manera jerárquica frente a un desastre que tuvo tan diversos impactos; parte de ello tuvo que ver con que la capacidad para responder tan rápida y efectivamente se debió a que nuevas formas de interacción y gobernanza se desarrollaron durante el movimiento Occupy.

BB. A la luz de lo ocurrido en los últimos dos años, ¿cree usted que la literatura respecto a las geografías del poder y la geografía política requieren desarrollar nuevos conjuntos de ideas y conceptos para entender e investigar el paisaje y el derecho a la ciudad?

DM. Creo que esto puede ser un artefacto de mis propias lecturas selectivas o algo por el estilo, no estoy necesariamente seguro que necesitemos nuevos conceptos o nuevas teorías. Pienso que necesitamos un debate más robusto en torno a ellas, por ejemplo si ves los argumentos de John Allen respecto al poder en una forma distribuida -que opino tienen mucho valor pese a que considero que no son cien por ciento correctos-, pienso que deben ser puestos en debate con gente que quiera entender lo que el Estado contemporáneo es, cómo funciona y por qué opera de la manera en que lo hace. Quizás algo de lo que planteas está sucediendo y no me he percatado, pero pienso que se necesita más debate mucho más robusto en torno al uso del poder, el ejercicio del poder y el rol del 
Estado. Hoy el neoliberalismo ya cumplió 40 años y Foucault tenía algunas de las respuestas en 1970, pero creo que hay mucho más por desarrollar. $\mathrm{Y}$ respecto a esto, pienso que no solo se necesita más debate o pensamiento teórico, sino que realmente se requiere más trabajo empírico. Pienso que el poder es más un asunto empírico que teórico, ya que se puede ver cuando se ejerce, de tal modo que preguntas como si está distribuido o centralizado u otras por el estilo pueden ser develadas y pensadas empíricamente, en lugar de solo hablar acerca de lo que nos parece podría ser. Preferiría ver algo de investigación realmente robusta respecto a los modos cambiantes de ejercicio del poder, pienso en los movimientos globales de 2011, desde Grecia hasta aquí, a los Estados Unidos, México y más allá; hay un montón de oportunidades para intentar dilucidar cómo operó el Estado, qué hizo, cómo operaron los movimientos sociales, qué hicieron, como se usó y ejerció el poder y qué relación tiene eso con diferentes tipos de espacios. Una de las cosas realmente buenas de Occupy Wall Street fue que demostró que algo que yo había tenido por cierto durante un largo tiempo estaba equivocado, cual es que no hay oportunidad para usos políticos del espacio público de propiedad privada. Yo había argumentado esto fuertemente, todo el discurso del fin del espacio público, que la privatización del espacio público estaba Ilevando hacia el fin de la política o de los usos políticos del espacio y Occupy probó lo contrario empíricamente, todavía pienso que parte de las discusiones teóricas son importantes y correctas acerca de las maneras en el poder se ejerce de modo diferente o cómo el espacio es controlado de otras maneras, pero empíricamente yo estaba equivocado y eso es realmente agradable, en este caso (risas).

DO. Respecto a las diversas formas de ejercer el poder, quería pedirle que nos contara sobre su investigación y punto de vista respecto a la doctrina del foro público ${ }^{5}$ en los Estados Unidos y de qué modo esta supresión

\footnotetext{
5 Ver Mitchell, D., 2003. The Liberalization of Free Speech: Or, How Protest in Public Space is Silenced. Stanford Agora, 4. Disponible en http:// agora.stanford.edu/agora/volume4/mitchell.shtml
}

sutil de la libre expresión está modificando el espacio público en cierto sentido.

DM. El dictamen original respecto a la doctrina del foro público, emitido en 1939, es muy interesante. En primer lugar, no concitó una mayoría en la Corte Suprema; había una pluralidad, de tal manera que la corte estaba muy dividida. Pero lo que declaró fue que los espacios públicos de la ciudad, parques y calles en particular, han sido dispuestos para el uso del público, de los ciudadanos -de hecho creo que hablan del pueblo-, con el fin de participar del discurso político incluyendo la protesta y ese tipo de cosas. Es un hito, en tanto por primera vez la Corte Suprema planteó que en los Estados Unidos tenemos el derecho a ocupar los espacios públicos por motivos políticos para la comunicación de ideas, sin embargo luego el dictamen establece que si bien ese derecho no puede ser restringido, ciertamente puede ser regulado. $Y$ esta regulación puede darse a partir de los criterios de tiempo, lugar $y$ modo; el tiempo para las actividades e interacciones, el lugar y el modo. Por otra parte, aparece un subconjunto de esto en lo que refiere a qué constituye discurso político y qué no constituye discurso político y eso es un elemento importante, aunque no necesariamente central para lo que discutiremos ahora. En los 30 años que siguieron al dictamen, la Corte Suprema comenzó a tomar el asunto tiempo, lugar y modo y cercar lo que era posible hacer en un espacio público por medio de la política, primero que todo decretando que el comercio y el movimiento de bienes, así como el movimiento de personas, en esencia sobrepasa o es más importante que el derecho a protestar, de tal manera que la protesta puede ser regulada para permitir el movimiento de bienes, lo que hace muy difícil por ejemplo organizar un piquete en una huelga. Y más tarde, la corte postuló una categoría muy amplia donde el discurso político podía ser regulado en consonancia con la "comodidad y el buen orden" -la frase dice algo así, que no es tan amplio como uno podría pensar. La corte también ha dictaminado a veces, ya desde los años 20, que el derecho más preciado de los hombres es el derecho a ser dejados en paz y allí están regulando algo respecto al espacio privado; sin embargo, la corte ha expandido esto al decir que tenemos el derecho a ser dejados en paz en 
el espacio público, lo cual es profundamente problemático porque implica que mientras puedo tener el derecho a hablar, no tengo el derecho a ser escuchado. Entonces, se puede regular la expresión si pone incómoda a la gente, ya que ellos tienen el derecho a ser dejados en paz y no escucharla. Todo esto termina resolviéndose en una serie de disputas espaciales acerca de dónde ocurre la protesta, a qué hora por supuesto, cuánto dura y cuánta gente congrega. La policía puede negociar la protesta, lo cual realmente les da la posibilidad de controlar a la gente y controlar la política, a través de estas leyes que permiten no ser oído, que permiten que el tiempo y forma de la protesta sean regulados. Luego, parte de mi argumento es que los movimientos sociales solo han tendido a ser efectivos cuando han estado dispuestos a romper con la ley, en directa desobediencia civil, mientras que por otro lado pueden ser muy poco efectivos; en los Estados Unidos durante los años 80 y 90, gran parte de las protestas no eran efectivas porque por ejemplo los arrestos se negociaban con antelación al acto. Entonces, la efectividad a menudo se obtiene rompiendo la ley o marchando sin un permiso en lugar de con él, que es la manera en la cual las protestas son gobernadas, en una dialéctica entre la necesidad de los protestantes de adherir a la ley porque a menudo existen buenas razones políticas para hacerlo y la necesidad de romper la ley. Esta dialéctica se había resuelto de manera continua hasta 1999 aproximadamente en favor de la policía y los gobiernos, de manera que los sociólogos hablaban de cómo desde principios de los años 70 al menos en Europa y Estados Unidos se había desarrollado lo que Ilamaron un sistema de gestión del orden público, que es un sistema negociado de protestas en lugar de tener a los manifestantes saliendo a la caIle, la policía golpeándolos, las cabezas rotas, etc. Esto llevó a un orden mucho mayor pero con la consecuencia de una gran inefectividad en la protesta. 1999 fueron las protestas contra la OMC en Seattle, todas las protestas entre entonces y el 11 de septiembre de 2001 e incluso un par más en 2002 y 2003 en alguna medida se salían del molde de este sistema de gestión del orden público y algo nuevo se estaba comenzando a inventar, algo más contencioso en un momento en que la policía tenía mucha más disposición a usar la fuerza otra vez. Se menciona a este sistema como una moderación del uso de la fuerza comparado con los 60 y 70, pero ahora parece haber una escalada de fuerza nuevamente y pienso que es otra razón por la cual, pensando en los Estados Unidos, el movimiento Occupy fue tan destacable en muchas formas porque en rigor desplazó el territorio de la protesta, en parte tomando un espacio y ocupándolo sin permisos ni negociación y en parte porque al hacerlo hicieron retroceder a la policía. Los gobiernos no sabían cómo responder y por eso les tomó dos meses antes de que hubiera una respuesta organizada al movimiento Occupy a través del país. Fue un momento interesante porque cambió la naturaleza de la protesta y el discurso de la política en los Estados Unidos al menos en un grado, y se dio en parte porque fue un tipo distinto de protesta, comparado tanto con la confrontación directa que emergió de Seattle como el sistema negociado que en muchos sentidos era todavía la norma.

DO. Sergio Rojas, filósofo chileno, sostiene que durante los últimos diez años es posible observar la emergencia de nuevos sujetos políticos cuya condición de tales se basa fuertemente en una aproximación estética a la subjetividad que luego deviene política. Rojas lo ejemplifica en la teatralidad que impregna muchas de esas manifestaciones, lo cual también pudimos ver el 2011. ¿Cuál es su reflexión al respecto?

DM. Sí, me parece realmente interesante. Creo que probablemente tiene razón, ahora que lo pienso. No solo aquí sino que por ejemplo las protestas de 2002 y 2003, cuando el G8 se reunión en Glen Eagle, Escocia, fueron altamente teatrales, con gente joven haciendo todo eso. Pero creo que también acierta en un sentido más amplio, ya que creo que la clave para cualquier tipo de protesta en relación con el espacio público es la visibilidad y a veces esa visibilidad implica grandes masas y números. Pero la otra forma de volverse visible es estando presente y haciéndose notar, de tal modo que las acciones teatrales son obviamente formas de Ilamar la atención. No estoy seguro respecto a la secuencia de la estética a la política, creo que están estrechamente ligadas, más que pensarlas de modo secuencial, pero de todos modos me parece un vínculo muy atractivo. No me imagino en la calle vestido de payaso o algo 
así, pero admiro a quienes lo hacen porque están inventando una nueva forma de política. Lo otro que esto genera, que fue cierto en Occupy y lo fue aquí y en muchos movimientos, es que trae un sentido del juego y la entretención a la política y las autoridades a menudo no saben qué hacer con eso. No saben cómo responder a ello y esto también tiene una historia larga: en los 60 durante el movimiento contra la guerra de Vietnam era común ver a chicas jóvenes sacar flores y ponerlas en los cañones de las armas, hacer cosas de ese tipo que son altamente teatrales, especialmente para los conscriptos de la Guardia Nacional que no tenían idea de cómo comportarse frente a eso. Pienso que esto es muy importante porque abre una vía diferente para entregar un mensaje que puede ser muy provocativo, pero no provocativo en un sentido violento.

DO. Esto se relaciona con lo que Beatriz decía respecto a la aproximación a la horizontalidad como un nuevo aspecto de la política. No creo eso sí que los estudiantes con quienes discutíamos el otro día piensen de ese modo. No sé si mucha gente tiene una idea muy elaborada o precisa acerca del Estado, pero rechazan la política tradicional en el sentido de la jerarquía de los partidos políticos y las formas establecidas de la política.

BB. No estoy segura si tensión es la palabra o más bien existe esta confusión entre estrategia y táctica, en el sentido que muchas de las preguntas y debates respecto al espacio público y el movimiento por la educación eran en torno a las tácticas; me parece que la racionalidad del movimiento podría impedir ese debate porque requiere disenso. No puede haber un $100 \%$ de acuerdo respecto a hacia dónde se quiere ir y debido a la diversidad del movimiento era más fácil enfocarse en las tácticas, como el teatro y otras.

DM. Pienso que la línea, el límite entre estrategias y tácticas es útilmente borrosa si piensan en la relación con lo alegre, como hablábamos antes, porque una de las cosas que muchos movimientos aquí y en otras partes están haciendo es modelar una forma diferente de estar en el espacio. Entonces, hay algo táctico en ello en términos de los movimientos de protesta. También hay algo de estratégico allí en tanto ellos están inventando nuevas formas de ser y nuevas formas de vivir y si bien puedo pensar en varias críticas a eso, por otro lado pienso que es una movida importante para sugerir que otro mundo es posible, si usamos ese eslogan. Para mí eso es estratégico en el sentido de pensar en nuevos modos de vida, y eso o parte de eso es decir que el Estado hace lo que el Estado hace pero nosotros de todos modos vamos a vivir de una manera diferente.

BB. Y usar el espacio público para hacerlo visible.

DM. Exactamente, y pienso que es realmente importante. No sé si es suficiente, pero probablemente es necesario. 